\section{Inhalte gemeinsam bearbeiten}

Wir möchten Ihnen in diesem Kapitel ganz unterschiedliche Dienste für die Zusammenarbeit an Textdokumenten (Texte, Tabellen, Präsentationen) vorstellen. Dabei ist das zuerst behandelte Etherpad für den schnellen Einsatz gedacht, die Office-2.0 Instrumente ermöglichen eine anspruchsvolle Bearbeitung von Text, Tabellen, Präsentationen u. ä., so wie man das von den Desktop-Office-Suiten her gewohnt ist. Am Ende des Kapitels gehen wir dann noch auf die Wikis ein, diese dienen eher dem längerfristigen Wissensmanagement als dem Dokumenten-Output.

\subsection{Etherpad}

Ein Etherpad einzusetzen, geht schnell und bietet kaum Schranken für Sie und Ihre Mitarbeiter/innen. Dafür ist es minimalistisch, bietet gerade einmal die Ablage von Text und Links und parallel eine Chatmöglichkeit, mit der man sich über den zu erstellenden Text verständigen kann. Ein Etherpad können Sie bei verschiedenen Providern ad hoc einrichten und schon geht es los!

Jede/r, die/der den Link zum bestimmten Pad hat, kann teilnehmen. Die Teilnehmenden werden durch Farben unterschieden, so dass ersichtlich ist, wer gerade die Ergänzungen erstellt hat. Hier ein Beispiel eines Pads aus dem bibcamp 2012:

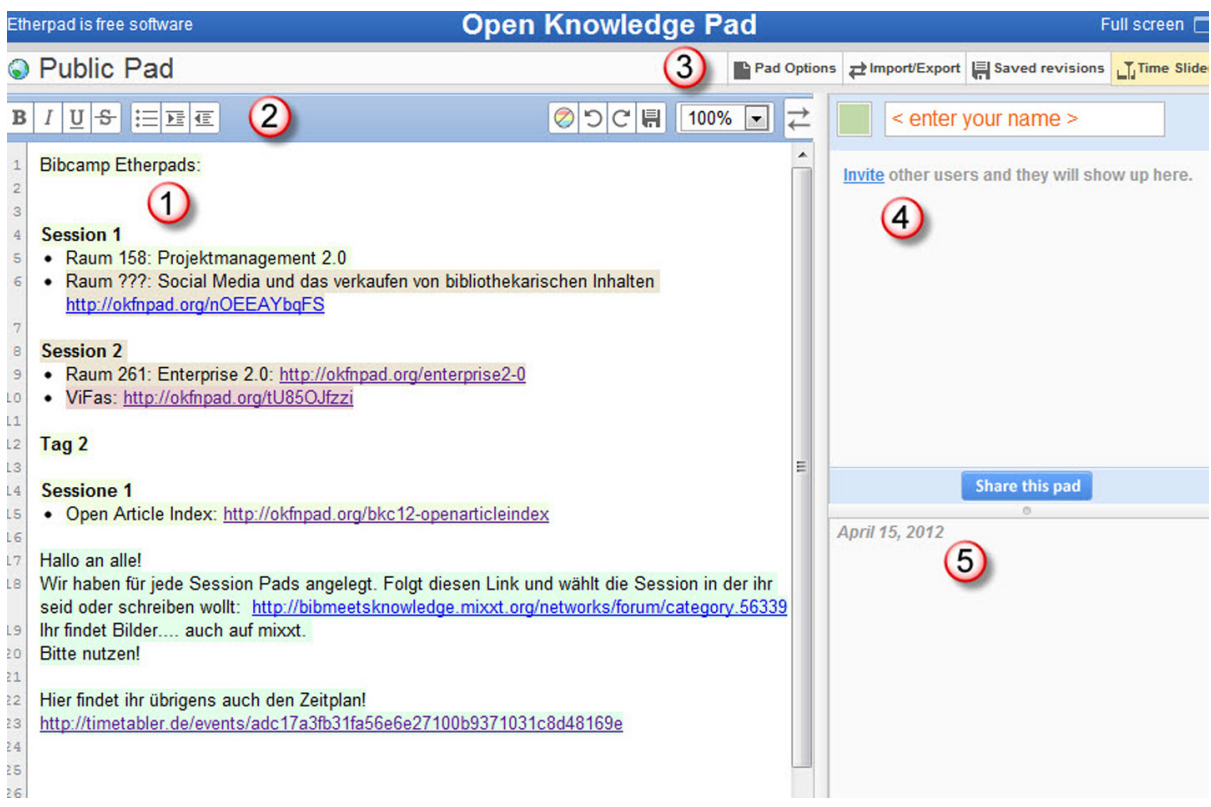

Das Textfeld (1) bietet einige wenige Auszeichnungsmöglichkeiten, die in der Leiste (2) auszuwählen sind. Auf der Leiste (3) sind grundlegende Funktionen inklusive Imund Export untergebracht, verschiedene Nutzer tragen sich im Feld (4) ein und bekommen verschiedene Farben zugewiesen. Ihre Beiträge im Textfeld (1) werden mit dieser Farbe unterlegt. Ebenso haben die Nutzer die Möglichkeit, unter (5) einen Chat zu führen als Metadiskussion. Zu mehreren Sessions des Bibcamps trugen mittels Etherpad auch Externe bei, die sich dann allerdings im Textfeld zurückhielten, aber im Chat Vorschläge machten.
Lernziele

In diesem Kapitel lernen Sie, verschiedene Dienste gezielt zur Koope-ration bei der Erstellung von Texten und Dokumenten einzusetzen. Inhalt dieses Kapitels:

- Etherpad

- Web-Office

- Wiki

Tipp

Webseiten, auf denen Sie ad hoc ein Etherpad einrichten können, werden bei der Etherpad Foundation aufgelistet [etherpad.org]

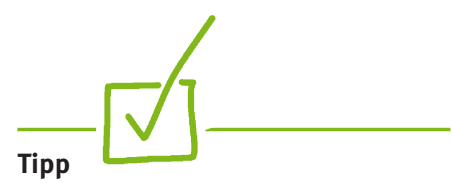

Nehmen Sie Etherpads immer dann, wenn Sie schnell etwas ablegen und diskutieren wollen, das später dann noch layoutet wird. Beispielsweise für

- Arbeitsgruppensitzungen unterstützen

- Sitzungen protokollieren

- Dokumentation/Methode von Diskussionen (alternativ zu Mindmaps) 


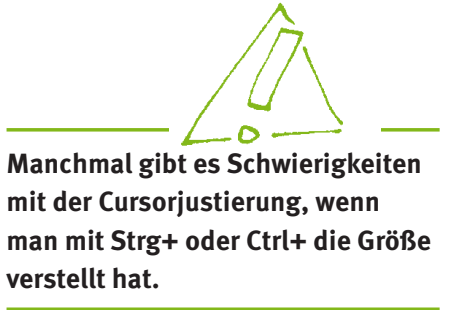

\subsection{Web-Office}

Die verschiedenen Web-Office-Suiten sind parallel zu den Angeboten zu sehen, die für einzelne Computer auf dem Markt sind, beispielsweise MS Office, Libre Office oder Open Office. Sie bieten verschiedene Büroanwendungen, vor allem anderen die Grundlagen Textverarbeitung, Tabellenkalkulation und Erstellung von Präsentationen. Angeboten werden jeweils die Grundfunktionen von Desktop-Office-Programmen wie beispielsweise die Suiten von Microsoft oder Libre Office, darüber hinaus jedoch die weitergehende Möglichkeit, dass mehrere an den Dokumenten, Tabellen oder Präsentationen zusammen arbeiten. Dies kann auch gleichzeitig geschehen!

\subsubsection{Google Docs/Drive}

Die Online-Office-Suite Google-Docs [docs.google.com] bzw. Google-Drive [drive. google.com] bietet vielfältige Möglichkeiten, für sich oder als Arbeitsgruppe Texte, Präsentationen, Tabellen, Formulare und Zeichnungen zu bearbeiten und abzulegen.

Der Nachteil besteht darin, dass alle einen Google-Account haben müssen, womit meist gleich die Debatte um Datenschutz und Akzeptanz von „Datenkraken“ beginnt. Zwar können Google-Dokumente auch ohne Anmeldung und Account bearbeitet werden, wenn sie frei im Netz stehen und über den spezifischen Link angesteuert werden können, dies ist dann aber wieder ein wenig öffentlicher als das Bearbeiten eines privaten Dokumentes.

Wenn Widerstände bei Teilnehmerinnen oder Teilnehmern bestehen, sollte man entweder auf das Office 2.0-Paket eines kleineren Anbieters wechseln (Zoho oder Thinkfree) oder ein Wiki einrichten (PBworks oder Wikispaces). Wobei zu bemerken ist, dass der in einem Wiki entstandene Text nicht wie ein Office-Dokument bearbeitet und ausgedruckt werden kann. Was in einem Wiki entsteht, ist also Text auf einer Webseite, nicht ein Dokument.

Wenn die Akzeptanz aber besteht, dann bietet Google-Docs gut anpassbare Instrumente, um entweder in einer geschlossenen Gruppe oder öffentlich im Web zusammen arbeiten zu können. 


\section{Die Google-Docs Startseite}

Öffnet man Google-Docs, öffnet sich zunächst die Startseite mit Ihrer Dateiverwaltung. Hier können Sie alle Ihre Dokumente und Ordner verwalten und neue erstellen. Neben den Formaten, die Google als Office-Produkte anbietet (Text, Tabelle, Formular und Zeichnung) ist es auch möglich, andere Dateien wie z. B. PDFs oder Bilder in Google-Docs abzulegen.

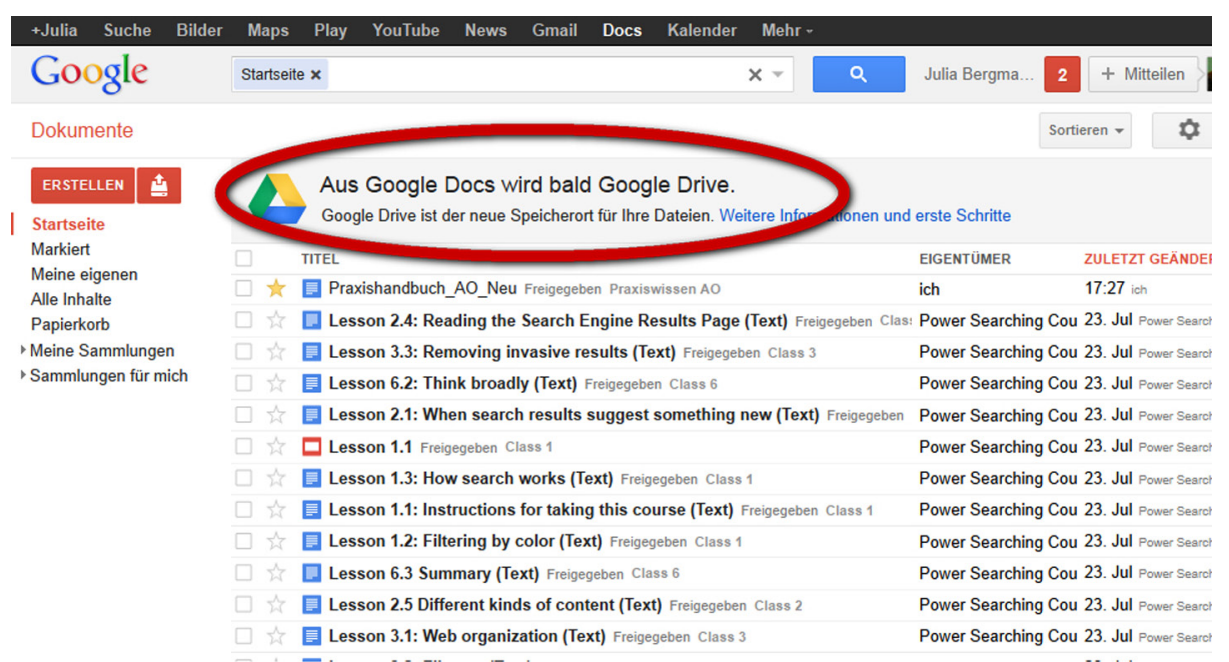

In der Übersicht sehen Sie auch, welches Dokument Sie mit anderen teilen und wer zuletzt daran gearbeitet hat. Sind seit Ihrer letzten eigenen Bearbeitung in einem Dokument Änderungen vorgenommen worden, wird dies dadurch signalisiert, dass der Titel des Dokumentes fett dargestellt wird.

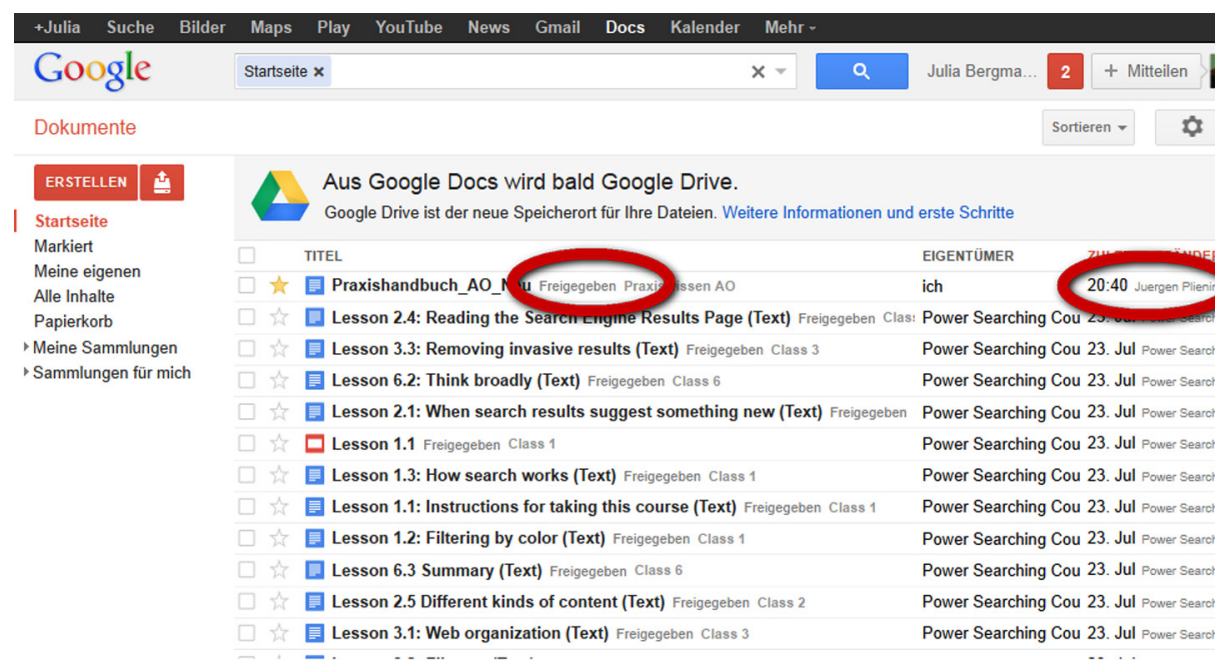

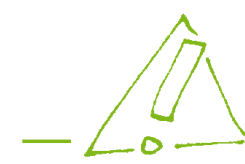

GoogleDocs wird derzeit zu GoogleDrive, welches zusätzlich eine leistungsfähige Verwaltung von Dateien mit Ablage- (bis zu 5 GB) und Zugriffsmöglichkeiten von den verschiedensten Internetgeräten bietet.

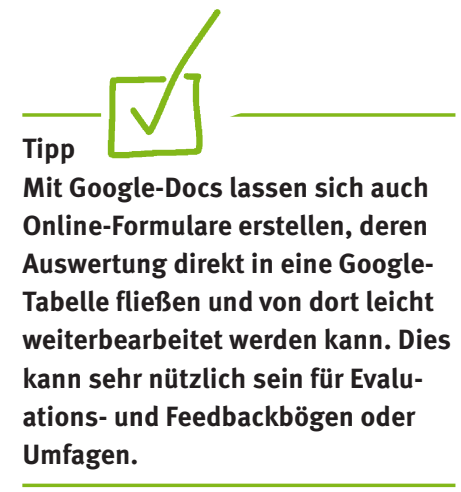


Sie können Ihre Dateien in Sammlungen/Ordnern organisieren. Dokumente, an denen Sie gerade arbeiten, können Sie auch mit einem Stern markieren, um das Dokument in Ihre Favoritenliste aufzunehmen und einen Schnellzugriff über die StandardSammlung „Markierte“ einzurichten.

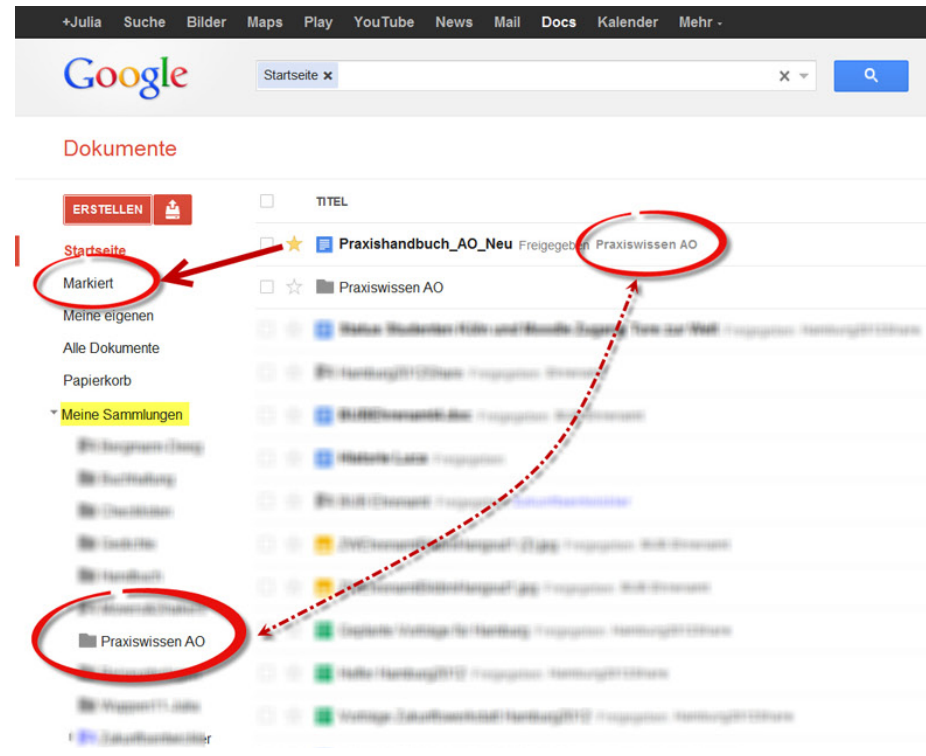

Um Dateien in Google-Docs zu bearbeiten, können Sie entweder über den Button Erstellen ein neues Dokument öffnen oder Sie können ein bereits begonnenes OfficeDokument in Google hochladen, um es hier abzulegen oder weiter zu bearbeiten.

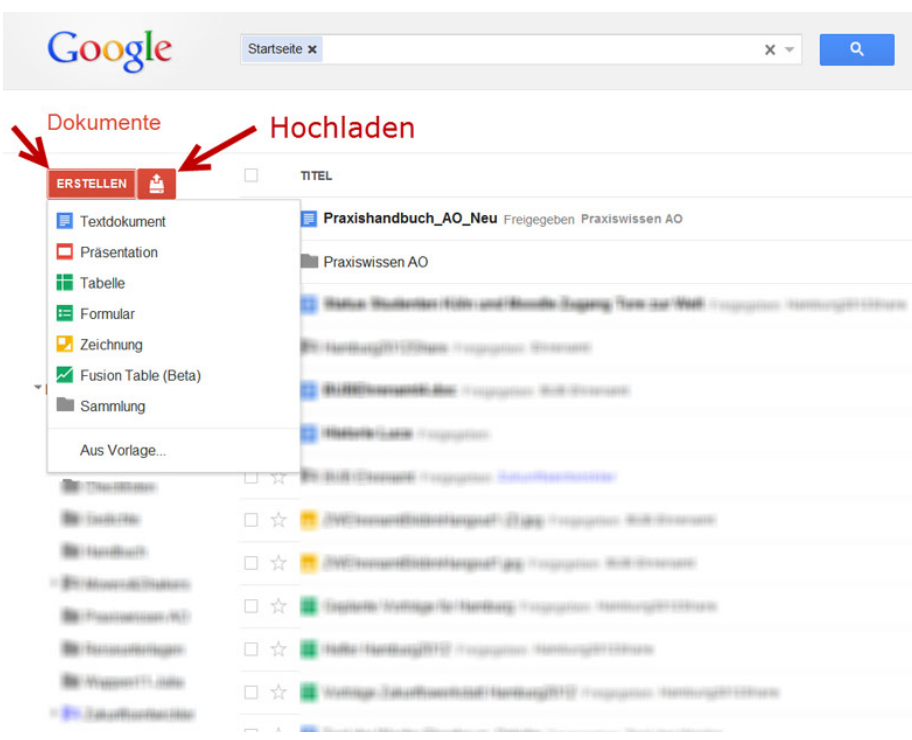


Wenn Sie ein Dokument hochladen, fragt Google, ob Sie das Dokument als OriginalDatei abspeichern möchten oder zur weiteren Bearbeitung in das Google-Docs Format konvertieren möchten.

Sie können sowohl Dokumente als auch Ordner für andere freigeben. Das Freigeben von Ordnern ist sehr nützlich, wenn Sie mit einer Gruppe von Personen mehrere Inhalte bearbeiten. Haben Sie einen Ordner für eine Gruppe freigegeben, werden diese Rechte automatisch auf alle Dokumente vererbt, die Sie in dieser Sammlung/Ordner ablegen. Sie müssen die Freigabe dann nicht bei jedem Dokument einzeln vornehmen.

\section{Bearbeiten von Dokumenten}

Google-Docs bietet neben den gängigen Office-Funktionen zum Formatieren eines Dokumentes (Text-Dokument, Tabelle oder Präsentation) nützliche Funktionen für das gemeinsame Arbeiten an Dokumenten. Dazu gehören die Freigabe von Dokumenten zur gemeinsamen Bearbeitung, Chatfunktion, die automatische Erstellung eines Überarbeitungsprotokolls und die Kommentarfunktionen. Diese Funktionen sorgen für eine einfache und klar strukturierte Zusammenarbeit an einem Dokument.

Bei Google ist es außerdem möglich, dass mehrere Personen gleichzeitig ein Dokument bearbeiten, was den kreativen Prozess bei der Formulierung besonders beflügeln kann. Bei längeren Texten ist dies zudem nützlich, weil jeder unabhängig vom anderen an seinem Textteil arbeiten kann, ohne von anderen „blockiert“ zu werden. Die Eingaben anderer werden für alle sofort sichtbar, so dass man jederzeit den aktuellen Stand sieht. Dabei werden die Cursors der jeweiligen Bearbeiter farblich unterschieden und können so leicht im Text erkannt werden.

\section{Ein Dokument für andere freigeben}

Wenn Sie ein Dokument geöffnet haben, dass Sie gern für andere freigeben möchten, klicken Sie oben rechts auf den Button freigeben.
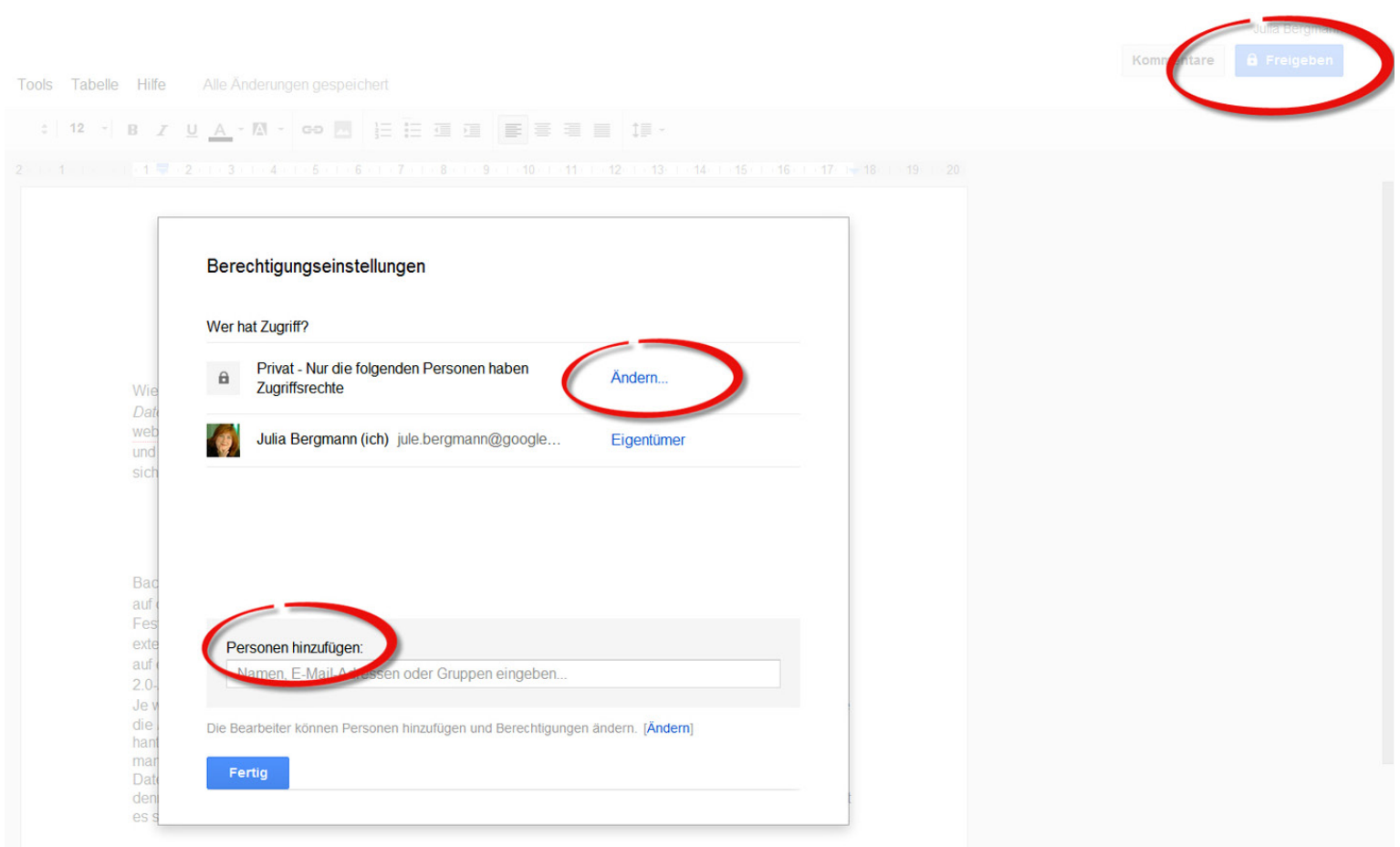
Hier haben Sie nun verschiedene Optionen für die Freigabe eines Dokumentes.

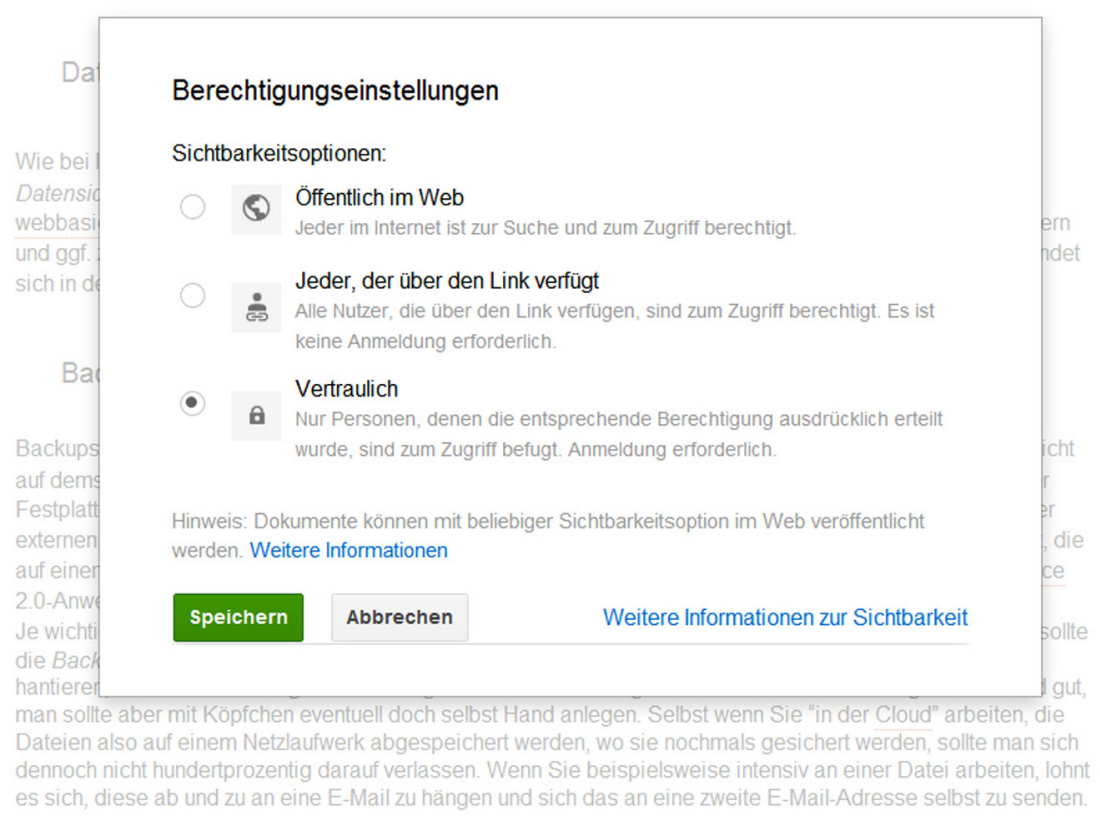

Vertraulich - Nur eingeladene Personen haben Zugriffsrechte

Bei dieser Option können nur Personen das Dokument öffnen, die von Ihnen explizit dazu eingeladen wurden.

Haben Sie Vertraulich ausgewählt, geben Sie im nächsten Schritt unter „Personen hinzufügen“ die E-Mailadressen der Bearbeiter ein, die Sie einladen möchten. Bei jedem einzelnen Bearbeiter können Sie festlegen, ob derjenige

- nur lesen

- kommentieren

- bearbeiten

darf.

Jeder, der über den Link verfügt

Hierbei wird ein Link generiert, den man mit anderen teilen kann. Dabei benötigen die Teilnehmer keinen Google-Account, um das Dokument öffnen zu können. Bei dieser Option gibt es zudem die Auswahl, ob die Personen, die das Dokument über den Link öffnen,

- nur lesen

- kommentieren

- bearbeiten

dürfen.

\section{Öffentlich im Web}

Bei dieser Option wird aus Ihrem Office-Dokument sozusagen eine Webseite, die mit Hilfe von Suchmaschinen im Netz gefunden werden kann.

Auch hier unterscheidet Google beim Zugriff wieder in:

- nur lesen

- kommentieren

- bearbeiten 


\section{Gemeinsam bearbeiten}

Schreiben Sie in einem Dokument, das Sie mit anderen teilen, und ein weiterer Bearbeiter öffnet das Dokument, erscheint sein Nutzername oben rechts. Jedem Bearbeiter wird vom System eine Farbe zugeteilt, die vor dem Namen angezeigt wird.

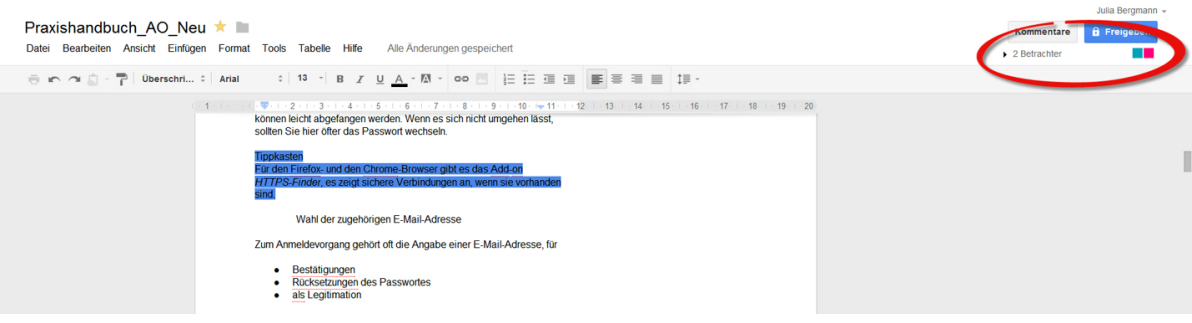

Im Text sehen Sie nun auch den farbigen Cursor des zweiten Bearbeiters und können verfolgen, was dieser ergänzt.

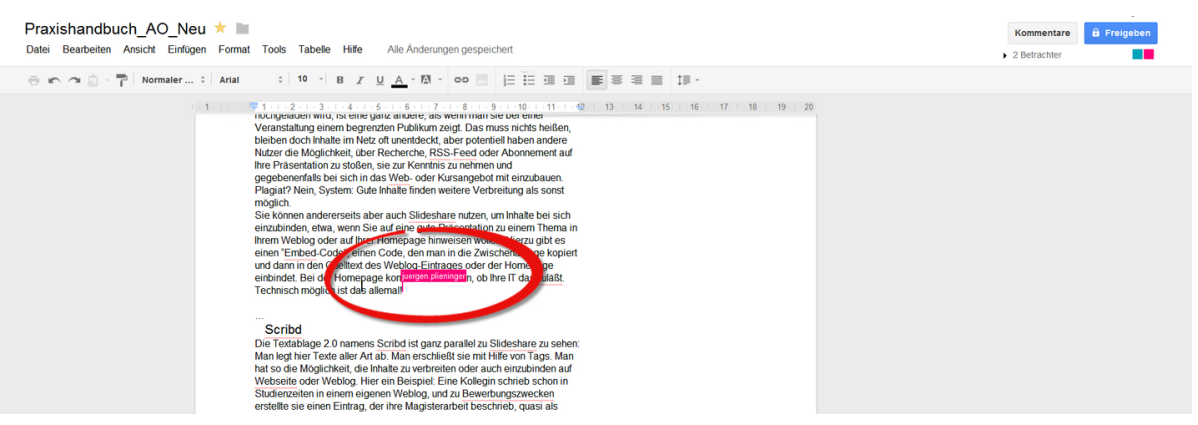

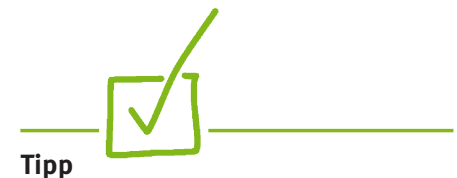

Sollten Sie mit mehreren Bearbeitern gleichzeitig an einem längeren Text arbeiten, und Sie wollen gezielt die Ergänzungen eines Bearbeiters live mitverfolgen, können Sie einfach oben rechts auf das Farbfeld vor dem Namen des Bearbeiters klicken, und die Ansicht springt automatisch an den Punkt im Dokument, wo dieser gerade Ergänzungen macht. 
Die Chatfunktion

Sie können Ihre Ergänzungs- bzw. Veränderungsvorschläge auch begleitend in einem Chat diskutieren. Dazu klicken Sie oben rechts auf „Betrachter“. Es öffnet sich nun an der rechten Seite ein Frame, in den Sie unten Ihre Chatnachricht eingeben können.

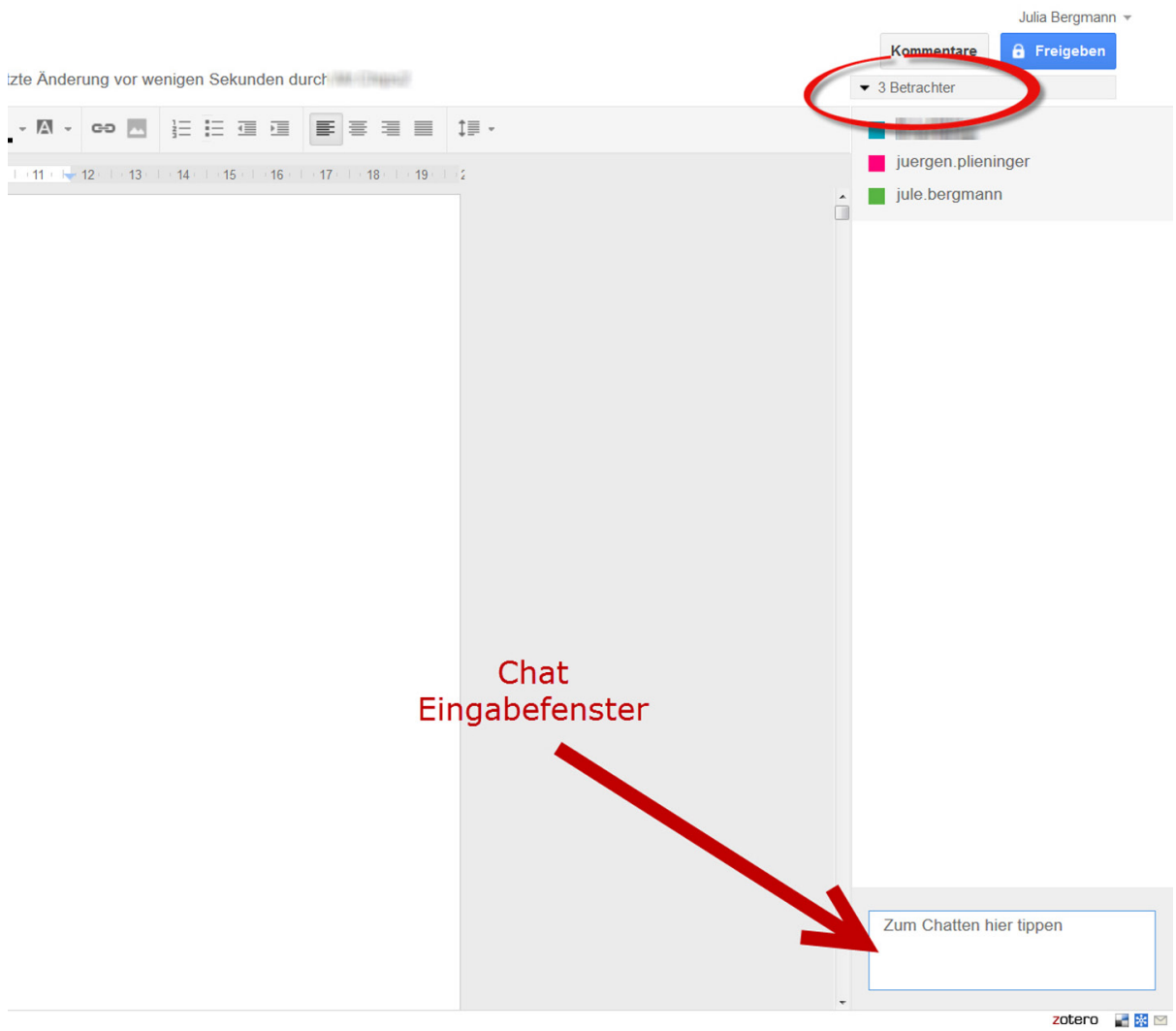




\section{Das Überarbeitungsprotokoll}

Um zu sehen, was von anderen Mitarbeitern seit Ihrem letzten Besuch verändert wurde, klicken Sie oben links auf Datei $\rightarrow$ Überarbeitungsverlauf anzeigen.

Der Überarbeitungsverlauf wird von Google automatisch erstellt, seine Aktivierung kann also auch nicht vergessen werden.

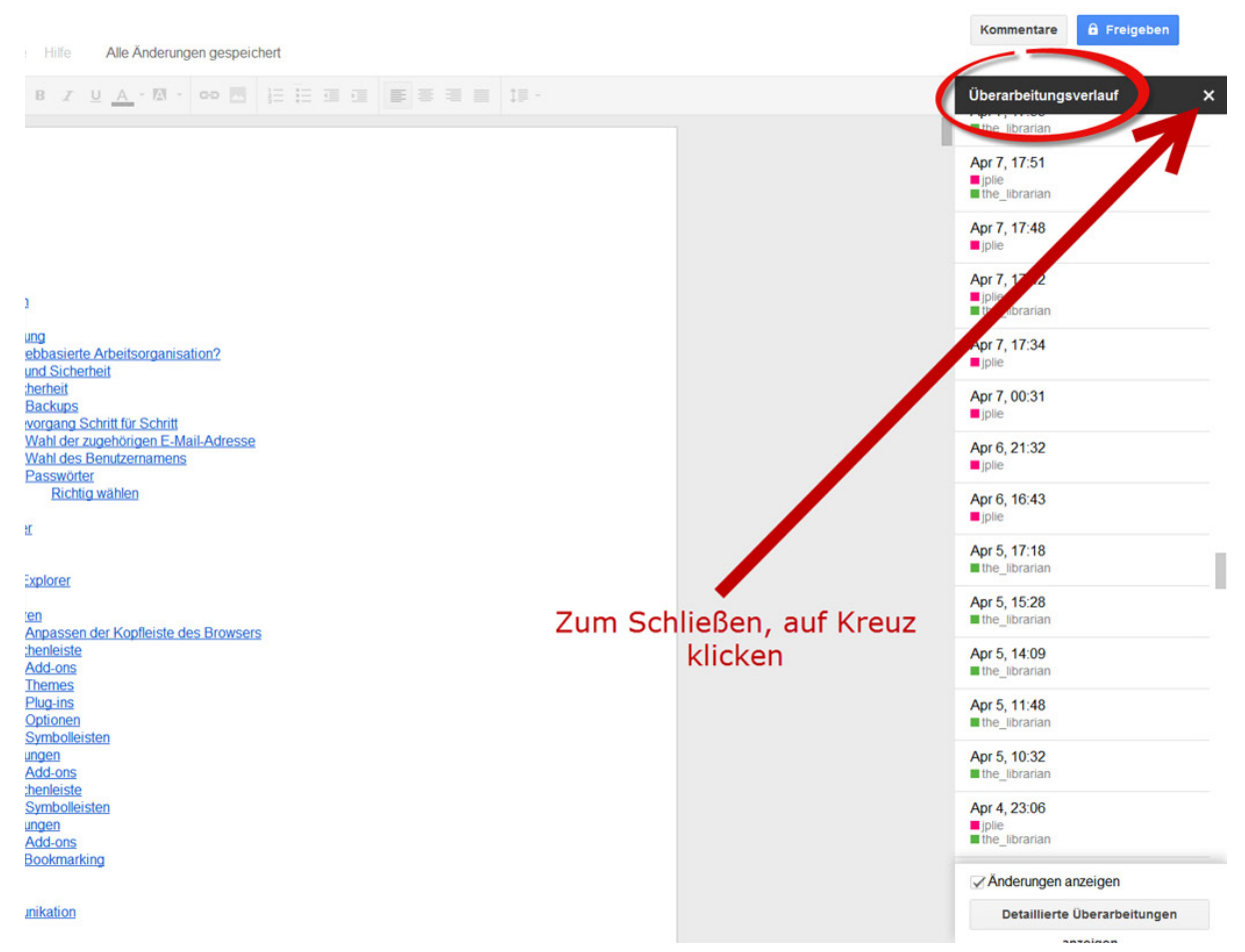

Rechts hat sich nun ein Frame geöffnet, in dem Sie den Veränderungsverlauf sehen können. Wer hat wann etwas geändert. Um sich diese Veränderungen im Dokument anzeigen zu lassen, wählen Sie die Änderungen aus, die Sie gern ansehen möchten. Diese werden dann im Dokument farbig markiert, wobei wieder jeder Bearbeiter eine Farbe vom System zugeteilt bekommen hat.

In der Ansicht des Bearbeitungsverlaufs können Sie nur lesen, aber nicht editieren. Um wieder in den Editier-Modus zu wechseln, schließen Sie den Überarbeitungsverlauf mit dem Kreuz oben rechts. 


\section{Die Kommentarfunktion}

Wichtig für die Zusammenarbeit an Dokumenten ist die Kommentarfunktion. Um einen Kommentar zu setzen, markieren Sie die entsprechende Textstelle und machen einen Rechtsklick. Es erscheint ein Kontextmenü, aus dem Sie „Kommentar“ auswählen.

Tippen Sie Ihren Kommentar und klicken zum Abschließen den Button „Kommentar".

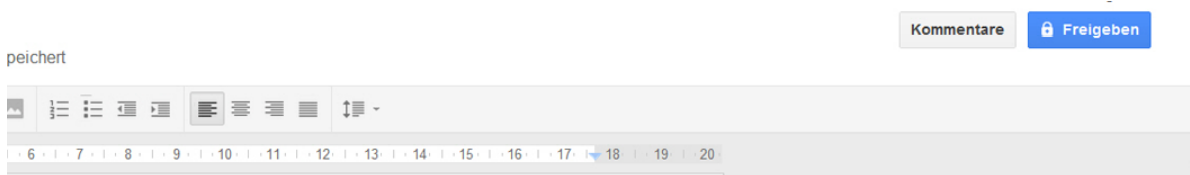

rbeit oder zu Hause, sollten Sie auch (wo möglich) eine regelmäßige online erstellten Inhalte durchführen. Ein Kriterium bei der Auswahl eines tie Möglichkeit, die online erstellten Inhalte durch eine einfache Routine sichern st umziehen zu können. Die Möglichkeit zum Export/Backup Ihrer Daten befindet Ingen bzw. Settings oder Optionen des jeweiligen Dienstes.

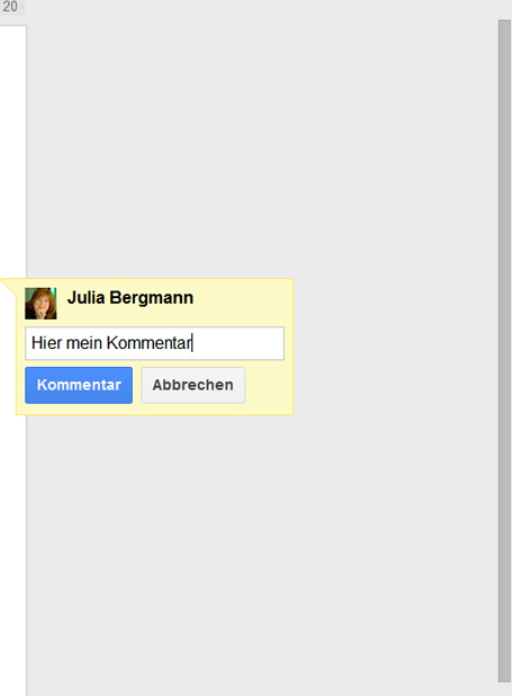

routinemäßig periodisch vornehmen Dabei sollten die Sicherungsdateien nicht it werden, sondern ortsunabhāngig verfügbar sein. Wenn die Daten auf Ihrer en dann sollte die Sicherung beispielsweise auf einem USB-Stick oder einer Laufwerk in der Cloud (vgl. Kapitel 10) sein. Wenn es sich um Daten handelt, die ispielsweise bei hier besprochenen Diensten wie Weblogs, Wikis oder Office I Sie sie auf die Festplatte herunterspeichern.

rbeit, die Sie gerade mit bestimmten Programmen erledigen, um so enger sollte iichts, wenn Sie gerade am Jahresabschluss sind und tagelang mit Excel öchentlich gemacht wird. Deswegen: Automatisierte Sicherungen schön und gut, entuell doch selbst Hand anlegen. Selbst wenn Sie "in der Cloud" arbeiten, die

Diese Textstelle ist nun gelb markiert und Ihr Kommentar erscheint rechts neben dem Text. Außerdem ist er sichtbar im Kommentarverlauf aller Kommentare, den Sie über den Button „Kommentare“ oben rechts aufrufen können.

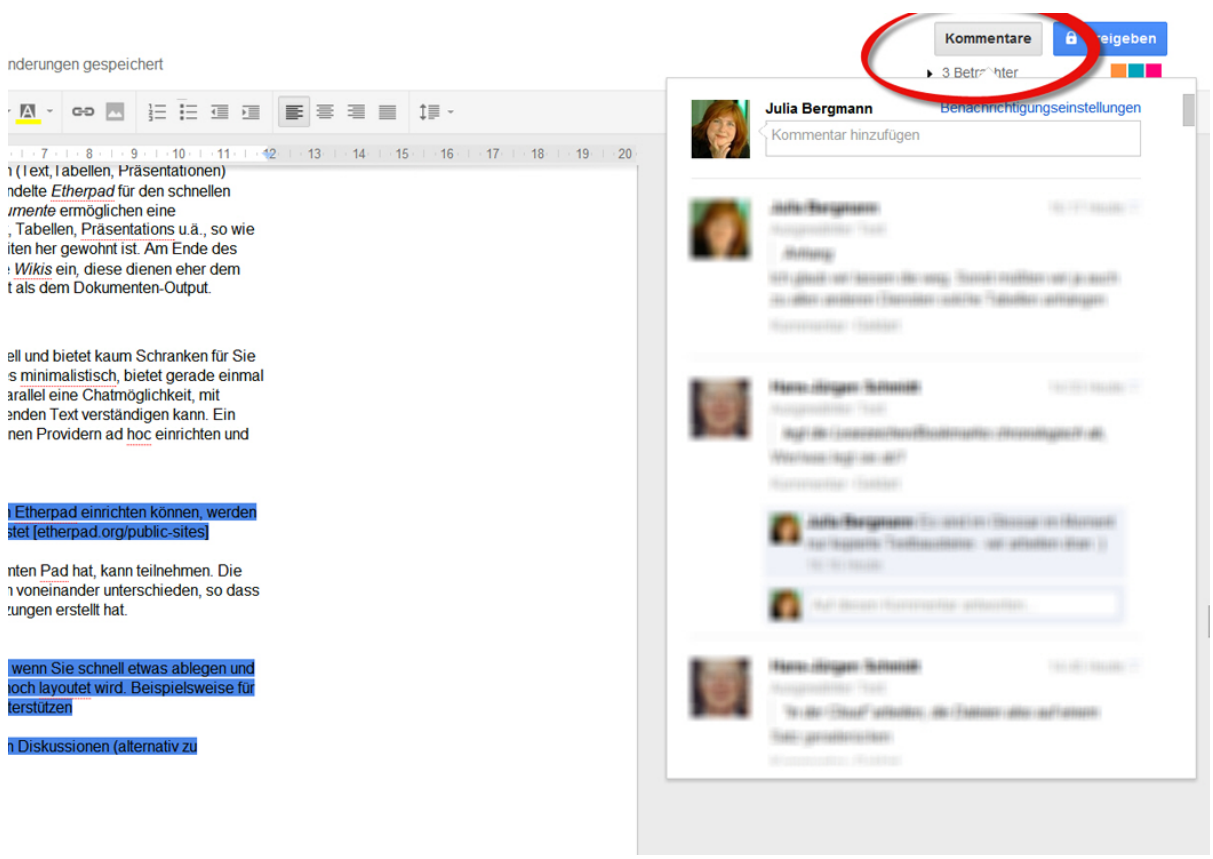


So können Sie schnell und übersichtlich allen Kommentaren zum Text folgen. Klicken Sie im Kommentarverlauf auf einen Kommentar, springt die Ansicht automatisch an die Stelle, wo dieser Kommentar gesetzt wurde.

Die anderen Bearbeiter des Dokumentes haben nun die Möglichkeit, Ihren Kommentar zu diskutieren. Wenn ein Sachverhalt geklärt ist, kann der Kommentar als „geklärt“ und somit geschlossen gekennzeichnet werden.

Bei Bedarf kann ein geschlossener Kommentar jederzeit wieder eröffnet werden.

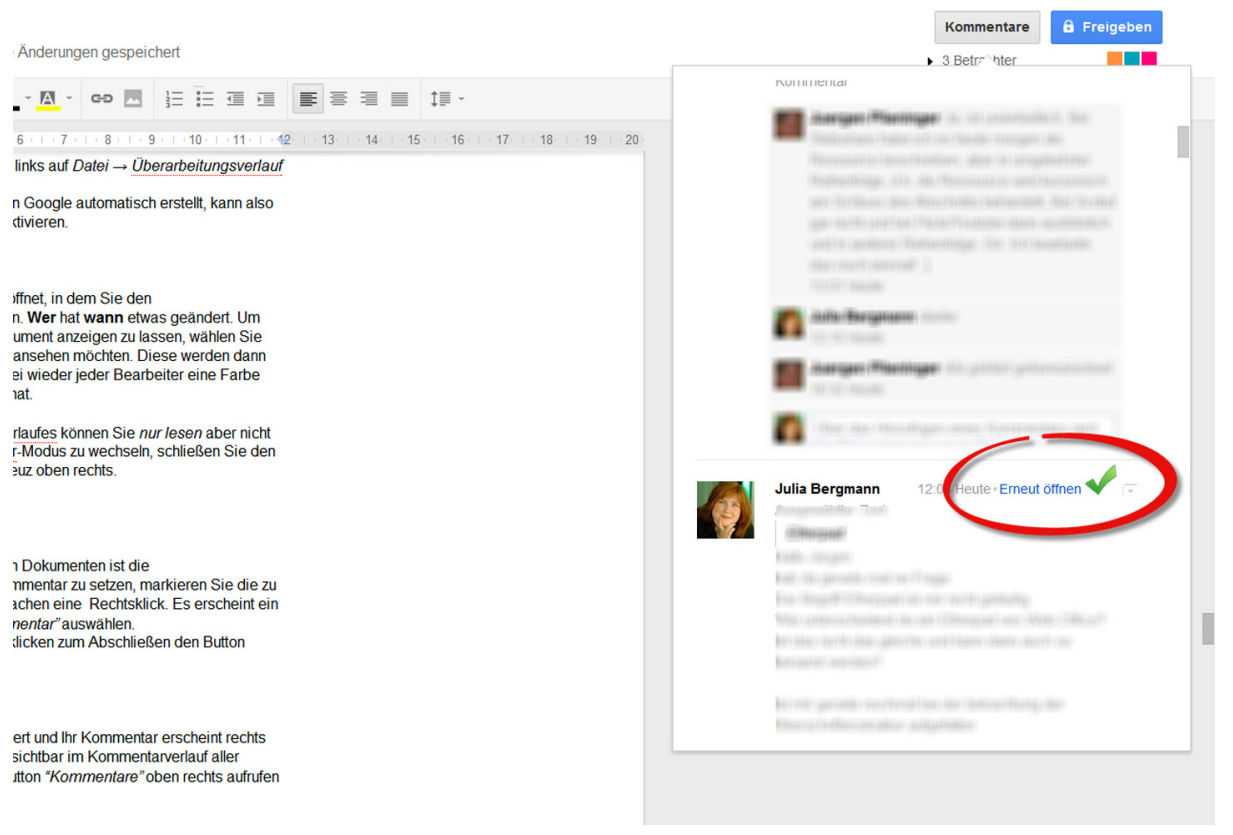

Sie können auch einen allgemeinen Kommentar über den Kommentar-Button eingeben. Er ist dann nicht an eine Textstelle gebunden.

\section{Alerting-Dienste}

Die Standardeinstellung für Benachrichtigungen ist so gesetzt, dass Sie über alle Vorgänge in den Kommentaren sofort per E-Mail benachrichtigt werden. Sie können diese Einstellungen aber auch ändern. Klicken Sie dazu auf den Button „Kommentar“ und klicken dann auf „Benachrichtigungseinstellungen“.

\section{E-Mail an alle Mitarbeiter}

Eine weitere Option, um mit den anderen Bearbeitern des Dokumentes zu kommunizieren, ist die Möglichkeit, an alle Bearbeiter eine E-Mail zu senden. Als E-Mailverteiler bietet Ihnen Google automatisch die E-Mailadressen aller Bearbeiter an, denen Sie das Dokument freigegeben haben. In einem Dialog können Sie daraus, durch das Setzen von Häkchen, eine Auswahl treffen oder an alle eine E-Mail senden.

Sie finden diese Funktion in Datei $\rightarrow$ E-Mail an Mitarbeiter senden. 


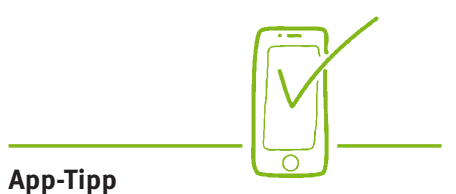

Für IOS und Android gibt es die App DocsToGo (Documents to go), die Ihnen das Bearbeiten und Rücksynchonisieren von Google-Dokumenten erlaubt. Die App kann auch mit anderen Cloud-Diensten wie z. B. Dropbox verbunden werden.

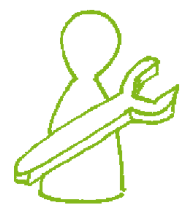

\section{Herunterladen von Dokumenten}

Zum Abschluss der gemeinsamen Arbeit an einem Dokument können sich alle Bearbeiter das Dokument im gewünschten Format herunterladen. Diese Funktion finden Sie unter Datei $\rightarrow$ Herunterladen als.

Hier stehen Ihnen, je nach Dokumentenart, verschiedene Formate zur Auswahl, so dass es kein Problem ist, wenn eine Person ggf. ein anderes Office-Produkt nutzt. Jeder kann hier das für seine Rechner-Installation günstige Format auswählen.

Sie können das Dokument natürlich jederzeit, auch zwischendurch, als Sicherung herunterladen. Oder Sie können die Datei am Ende ggf. auf dem Rechner nachbearbeiten, um z. B. eine Formatvorlage zu hinterlegen.

\section{Welche Anbieter gibt es im Bereich von Office 2.0}

- Google-Docs/Google-Drive: Wir haben oben die Textverarbeitung eingehend vorgestellt, doch finden Sie unter Google-Docs/Google-Drive die Möglichkeit, Tabellen, Präsentationen, Formulare, Zeichnungen und anderes anzulegen, gemeinsam zu bearbeiten und auch öffentlich weiter zu verwenden, beispielsweise als Dokumente oder Umfragen im Netz.

- Zoho [www.zoho.com]: Bietet eine sehr umfangreiche Auswahl an Web 2.0-Produktivitätstools, die alle in einer Grundfunktion kostenlos zu verwenden sind. ZohoWriter bietet Textverarbeitung, ZohoSheet die Tabellenkalkulation, ZohoShow die Präsentation, ZohoWiki ein Wiki etc. Wenn man an Projektmanagement interessiert ist, gibt es ZohoProjects, mit dem man gut für eine abgeschlossene Gruppe eine Projektumgebung mit Zeitplanung, Kommunikation, Dateiablage etc. aufbauen kann.

- Thinkfree [www.thinkfree.com] bietet ebenfalls Textverarbeitung, Tabellenkalkulation und Präsentationsmöglichkeit, ist in der Handhabung langsam und umständlich, bietet aber die Option des Offline-Bearbeitens von Dokumenten.

- Microsoft bietet unter Windows Live den Sky Drive [www.windowslive.de/Skydrive/] an, auf welchem man Office-Dokumente hochladen und mit anderen gemeinsam bearbeiten kann. Für Geschäftskunden wird gerade unter Office 365 [www.microsoft.com/de-de/office365/] eine kostenpflichtige Möglichkeit der Kollaboration auf einer Office 2.0-Plattform angeboten.

\subsection{Wiki}

Ein Wiki ist eine schnelle Erstellung von miteinander verknüpften Webseiten mit der Option, Dateien und Multimedia-Dokumente (Bilder, Audiodateien und Videos) auf diesen Seiten einbinden zu können. Und man kann diese Webseiten gemeinsam bearbeiten, wie bei der Wikipedia, dem besten Beispiel für ein funktionierendes Wiki. Bei vielen Wikis ist auch eine Rechteverwaltung möglich, so dass Sie abgeschlossene Webpräsenzen - beispielsweise für Arbeitsgruppen oder Kurse - einrichten können.

Die Software, die die Einrichtung und den Betrieb eines Wikis gewährleistet, ist oft kostenlos zu bekommen. Man kann sie auf einem Server installieren, anpassen und selbst administrieren. Wenn der Aufwand zunächst gering erscheint, so ist doch die Anpassung recht aufwändig und die Präsenz oft spamgefährdet. Deshalb empfehlen wir, einen Provider zu nehmen, an den Sie quasi den Aufwand für Einrichtung und Betrieb outsourcen. Das ist auch kostenlos möglich!

\subsubsection{PBworks: Schritt für Schritt}

PBworks ist ein US-amerikanischer Provider, der sich insbesondere auf den Bildungssektor spezialisiert hat. Ursprünglich hieß er pbwiki, das „pb“ stand für „peanut butter" und war eine Marketingidee: Schon der Firmenname sollte signalisieren, dass 
ein Wiki einzurichten so schnell geht, wie ein Erdnussbutterbrötchen zu schmieren. Allerdings will pbworks Ihnen etwas verkaufen, weswegen der Weg zu kostenlosen Wikis stets etwas schwer gemacht wird. Wir wollen es Ihnen daher Schritt für Schritt zeigen:

\section{Schritt 1: Registrieren}

Sie laden die Webseite [pbworks.com], wählen dann oben rechts „Login“, klicken hinter „no account?“ das blaue „sign up“ an und geben Ihren Namen, eine E-MailAdresse, die mit dem Account verknüpft ist und zum Schluss noch ein Passwort an. Wenn Sie dann „Create Account“ anklicken, wird Ihnen eine Bestätigungs-E-Mail zugesandt, bei der Sie einen Link anklicken müssen. Dann besitzen Sie bei pbworks einen Account.

\section{Schritt 2: Ein Wiki anlegen}

Wenn Sie sich mit Ihrem Account einloggen, werden, wenn Sie schon Mitglied bei Wikis von pbworks sind, die „Workspaces“ angezeigt. Rechts ist ein Feld, in welchem Sie Zugang zu bestehenden Workspaces abfragen können, und darunter befindet sich ein unscheinbarer Link „Create a new workspace“. Den klicken Sie an!

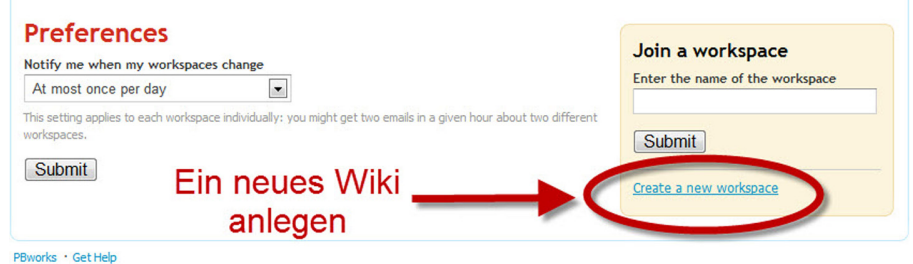

Dann müssen Sie einen geeigneten Namen für die URL des Wikis finden, einen Namen, der noch nicht verwendet wurde, bestätigen, dass das Wiki im Bildungsbereich eingesetzt und für nichtkommerzielle Zwecke verwendet wird (Sie sollten sie also nicht für kostenpflichtige Webinare verwenden!) und sind dann „drin“.

Einmal vergebene Webadressen für PBWorks-Wikis bleiben auch dann vergeben, wenn Sie den Wiki später wieder löschen. Richten Sie also möglichst nur dann einen Wiki ein, wenn Sie ihn auch verwenden möchten.

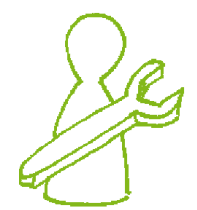




\section{Schritt 3: Die Hauptseite gestalten}

Die Hauptseite des Wikis, die Sie jetzt sehen, ist mit einem englischen Anleitungstext versehen. Vielleicht nicht die sinnvollste Gestaltung?

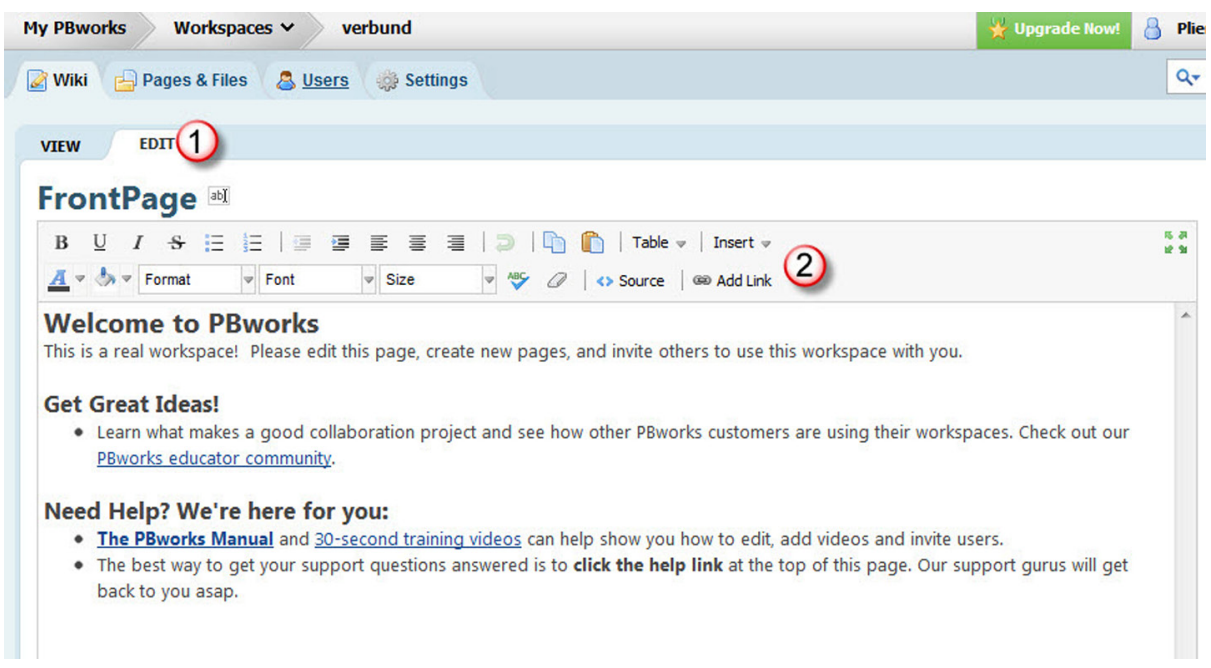

Klicken Sie die Registerkarte „Edit“ (1) an, schieben den englischen Text erst einmal nach unten und fügen Sie eigene Inhalte wie zum Beispiel eine Bildleiste oben ein und schreiben einen Willkommenstext. Sie können auch auf die Navigation auf der rechten Seite hinweisen. Wenn Sie Edit angeklickt haben, sehen Sie eine Editierleiste (2), wie Sie sie von der Textverarbeitung her kennen. Sie können also im Textfeld frei Text eingeben, einfügen und editieren und die Änderungen zum Schluss unten auf der Seite mit „Save“ speichern.

\section{Schritt 4: Dateien hochladen und verknüpfen}

Wir sprachen davon, ein Bild einzubinden. Zuerst einmal müssen Sie es als Datei hochladen. Hierzu klicken Sie auf die Registerkarte „Pages \& Files“ (1) und dann auf „Upload files“ (2) und können so Ihrem Wiki Dateien hinzufügen.

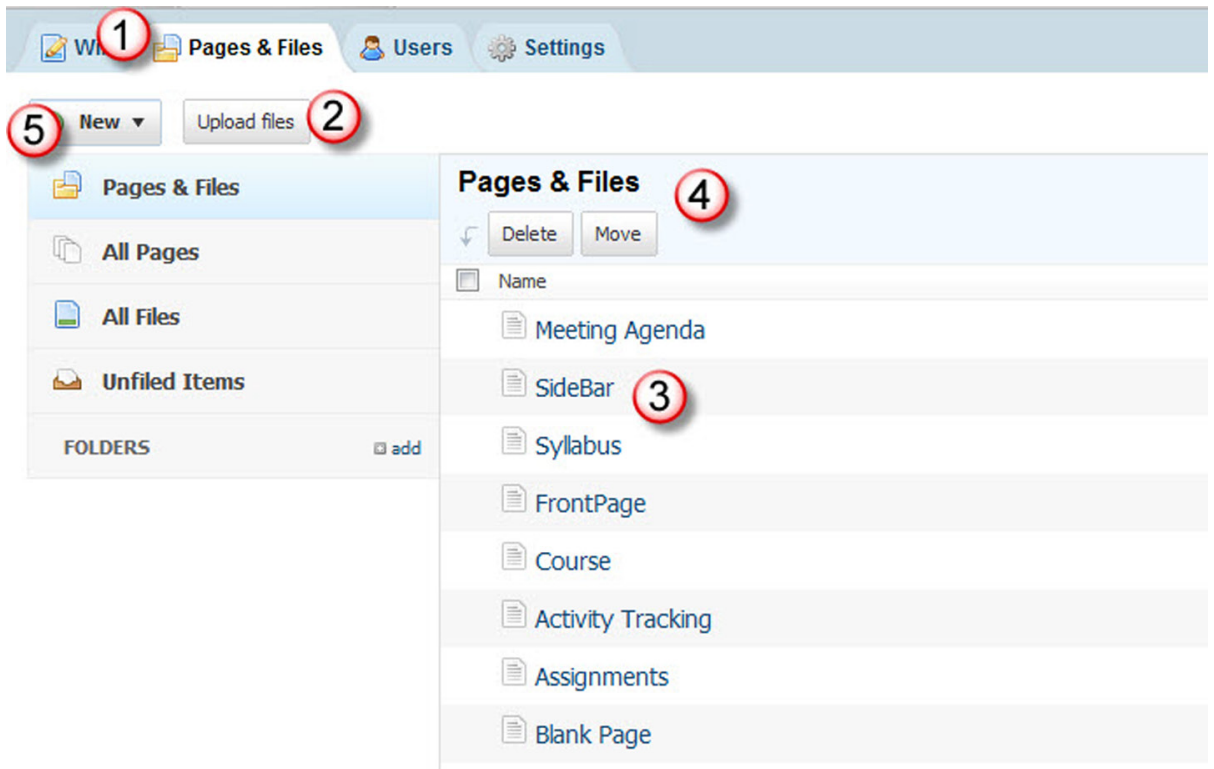


Unter (3) sind die Dateien aufgeführt, die schon im Wiki vorhanden sind, sie können gruppiert oder gelöscht (4) werden. Mit dem Knopf „New“ (5) können Sie Ordner anlegen und so die Dateiablage strukturieren.

Um jetzt einer Seite ein Bild hinzuzufügen, wechseln Sie wieder zurück auf ,Wiki“ (1), öffnen die Bearbeitung wie vorhin mit „Edit“ (2) und öffnen rechts beim Fensterchen „Insert links“ (3) die Registerkarte „Images and files“ (4). Hier bekommen Sie eine Liste der hochgeladenen Dateien angezeigt (5) und können durch einfaches Anklicken - Festhalten - In-die-Seite-Ziehen das entsprechende Bild einfügen und positionieren. Optional können Sie auch Bilder per URL (6) einbinden.

Die Größe des Bildes lässt sich anpassen, indem Sie das Bild anklicken und die Markierungen an den Ecken des Bildes mit der Maus anfassen und bewegen. Ziehen Sie möglichst diagonal, dann bleiben die Seitenverhältnisse des Bildes bestehen.

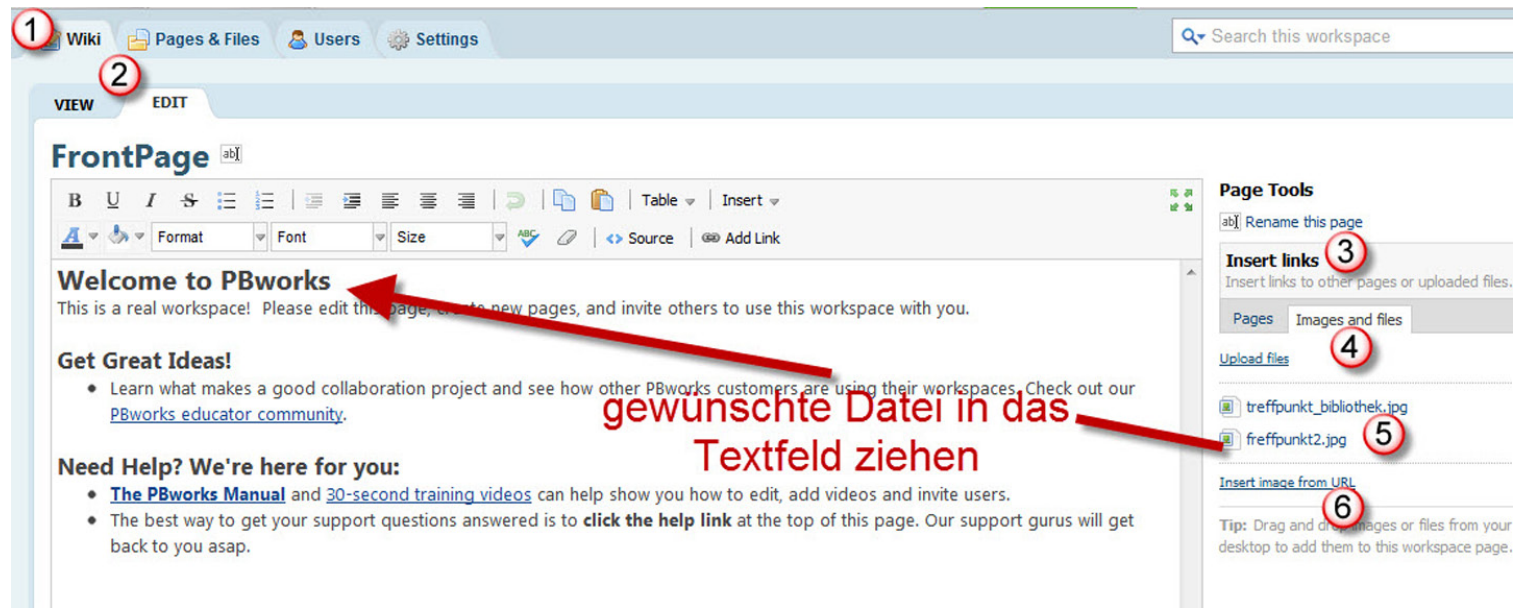

\section{Schritt 5: Unterseiten anlegen und verknüpfen}

Seiten anlegen geht ebenso leicht: Rechts „Create a Page“ (1) anklicken, dann wird eine Seite geladen, wo Sie den Seitennamen und eventuelle Zuordnung eingeben können (2). Bestätigen (3) und fertig!

\begin{tabular}{l}
\hline Wiki Pages \& Files 8 Users 19 Settings \\
Create a new page
\end{tabular}


Wenn Sie eine Seite bearbeiten und auf eine andere Seite verlinken wollen, markieren Sie die entsprechende Textpassage, drücken auf das „Add Link“-Zeichen mit der Verknüpfungskette, bekommen ein kleines Pop-up-Fenster und können hier mit der entsprechenden Seite verknüpfen.

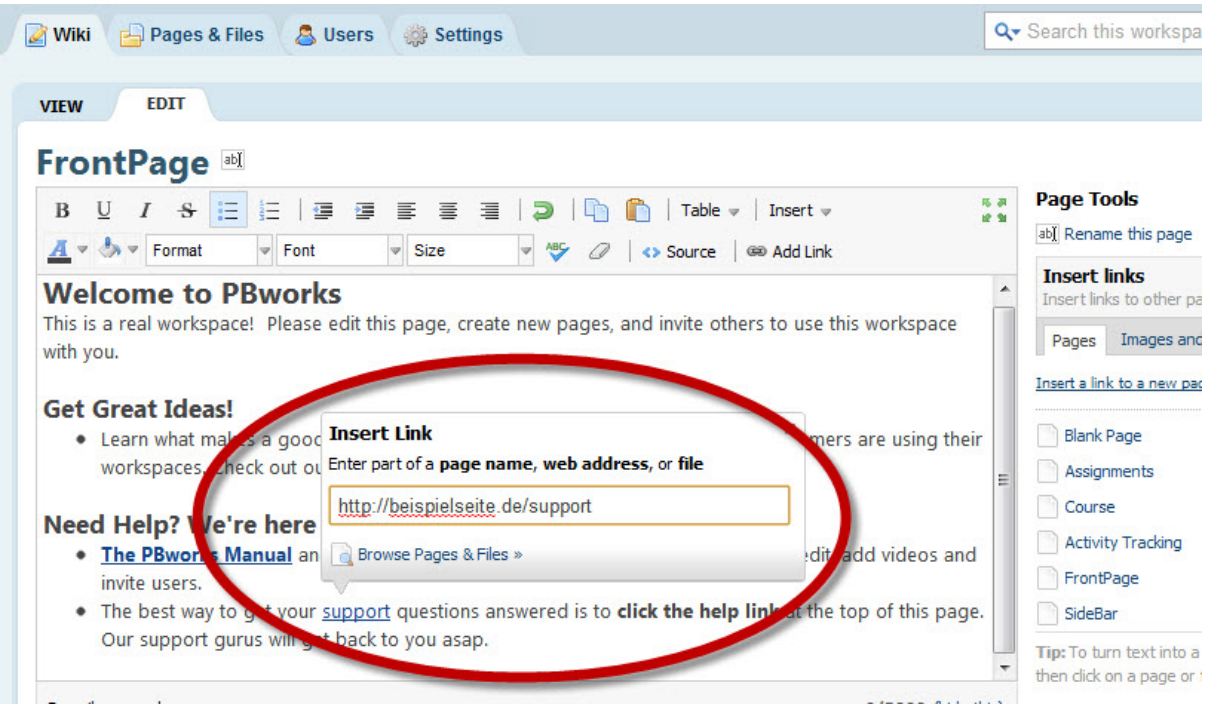

\section{Schritt 6: Eine Navigation erstellen}

Ursprünglich waren Wikis wild wachsend gedacht: Inhalte vielfältig ohne Hierarchie nebeneinander im Wiki einpflegen (rhizomartig) und miteinander verknüpfen, assoziativ, ohne Hierarchie! - In der Praxis ist das alles Schall und Rauch, der Benutzer verlangt nach klarer Struktur. Deswegen wird links die „Sidebar“ angeboten, in der man auf wichtige Seiten, Verzweigungsseiten oder eben auf eine hierarchische Struktur verlinken kann. Wenn Sie „Sidebar bearbeiten“ anklicken, sehen Sie, dass Sie die Sidebar ebenso bearbeiten können wie andere Wikiseiten auch. Sie können hier die wichtigsten Seiten des Wikis oder auch Verzweigungsseiten auflisten und verlinken. Ein Beispiel:

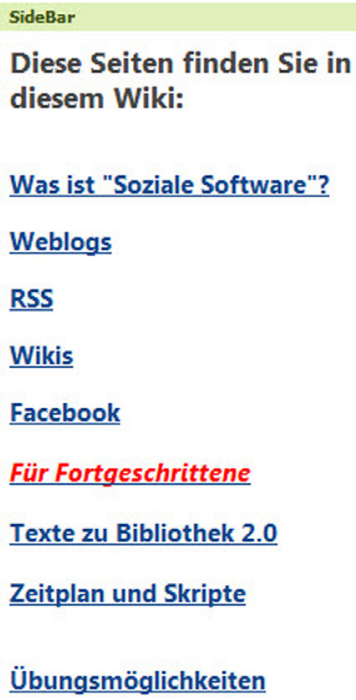




\section{Schritt 7: Benutzer hinzufügen und Rollen vergeben}

Sie sehen oben das Registerblatt „Users“: Hier können Sie Einladungen versenden, Benutzer verwalten und die verschiedenen Rollen zuweisen.

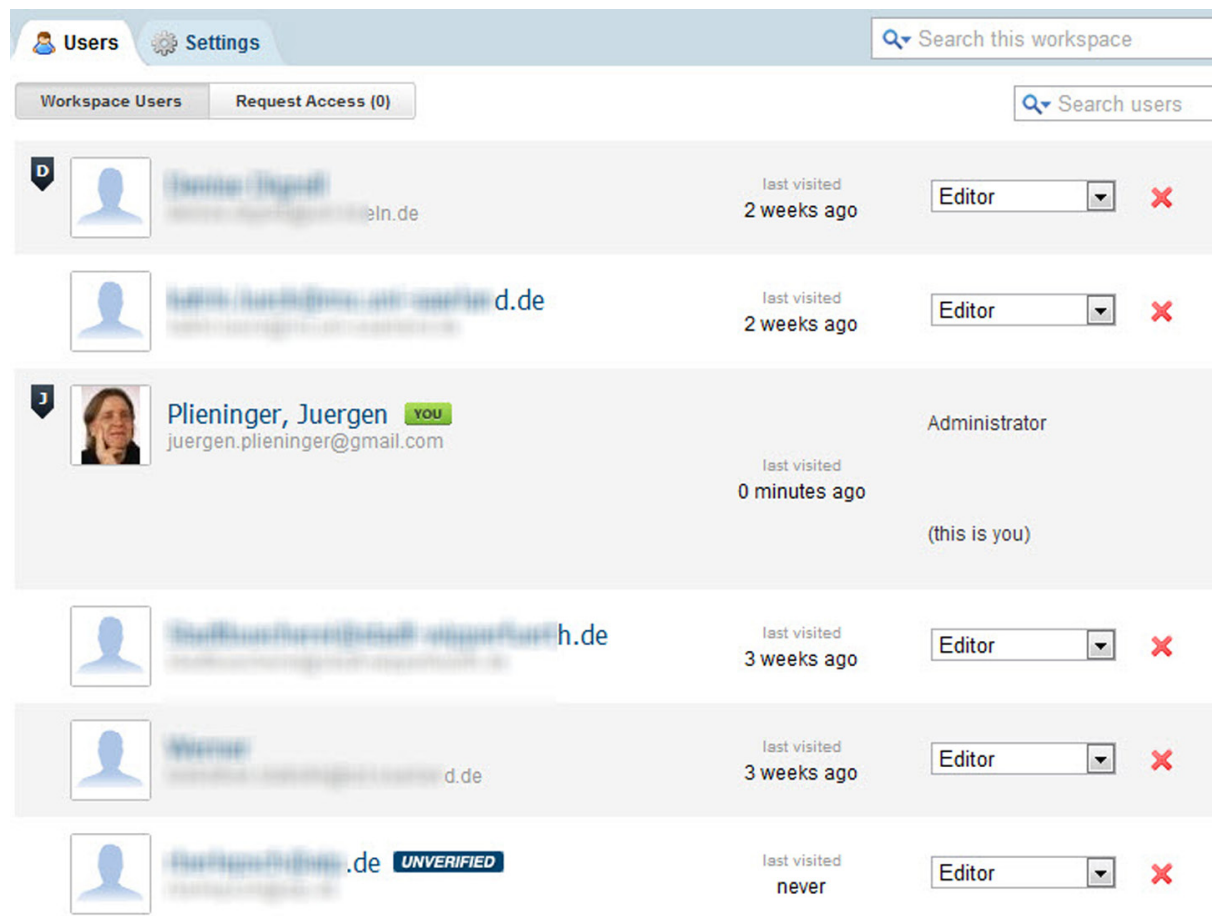

Über die verschiedenen Rollen gibt es im Hilfetext eine schöne Tabelle, auf der man nachvollziehen kann, was die einzelnen zugelassenen Benutzer dürfen und was nicht.

Und endlich in den „Settings“ können Sie einstellen, ob Thr Wiki frei ist und unter der spezifischen Webadresse von jeder/jedem gefunden werden kann oder ob es geschlossen und nur zugelassenen Benutzern zugänglich ist.

Ähnlich wie Pbworks funktionieren auch Google Sites und Wikispaces. Während das Google-Produkt völlig kostenlos zu nutzen ist, bieten PBworks und Wikispaces nur für den Bildungsbereich kostenlose Wikis an, die man auch mit verschiedenen Rechten für die Teilnehmer ausstatten kann - Voraussetzung für die Einrichtung eines abgeschlossenen Kursraumes, in dem die Diskussion geschützt stattfinden und man auch Dateien hinterlegen kann, ohne dass sie gleich alle Welt zur Kenntnis nimmt.

\subsubsection{Der Einsatz von Wikis}

Nachdem wir nun die Wikis technisch vorgestellt haben, möchten wir noch gerne auf ihre Einsatzgebiete eingehen. Sie sind außerordentlich anpassbar an die unterschiedlichsten Zwecke.

Wir haben Wikis hier in das Kapitel „Inhalte gemeinsam bearbeiten und erstellen“ eingeordnet, deshalb möchten wir - auch im Vergleich zu den weiter oben behandelten Office 2.0-Programmen - hierauf besonders eingehen. Sie können mit den Online-Office-Programmen schnell Dokumente erstellen, bearbeiten und auch einsetzen, die nah an der Konfiguration der bekannten installierten Office-Suiten liegen.

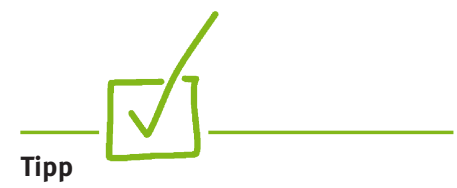

Wenn Sie einen (vergleichenden) Überblick über die Funktionalitäten verschiedener Wikisoftware bekommen möchten, ist Wikimatrix [www.wikimatrix.org] das Richtige für Sie: klicken Sie links die Wikisoftware an, die Sie interessiert, klicken Sie unten oder oben auf "compare" und es wird Ihnen in einer Matrix die Performanz der ausgewählten Systeme dargestellt.

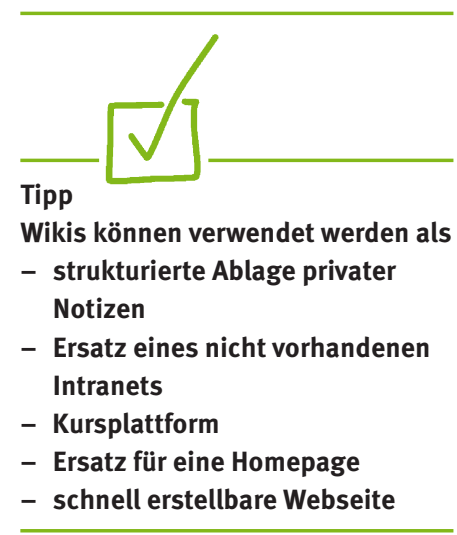


Darin liegt ihre Stärke: Sie sind schnell geöffnet, die Kollaborateure rasch eingeladen und alle können gleich loslegen, wenn sie einen entsprechenden Account haben. Auch das Exportieren geht schnell und man hat zuverlässig ein bestimmtes Format (Word-Datei mit .doc-Endung, Tabellenkalkulation mit xls-Endung und Powerpoint mit .ppt-Endung), das kompatibel ist mit dem, womit alle arbeiten.

Ein Wiki ist anders, es ist zwar schnell aufgesetzt, man muss sich aber Gedanken über die Gestaltung und Struktur machen. Die Einladung an Kollaborateure ist schnell erstellt, man hat aber ggf. mehr Aufwand, sie an das Arbeitsinstrument Wiki heranzuführen. Und endlich kann man zwar Inhalte immer schnell mit copy \& paste in eine Textverarbeitung übertragen, ein Export als komplette Word-Datei aber ist nicht möglich. Sprich: Office 2.0-Programme sind ein schnell einsetzbares und gut anpassbares Mittel, während Wikis eher für den strukturierten und langfristigen Einsatz geeignet sind, Zwecke, bei denen es auch auf Dokumentation und Archiv ankommt.

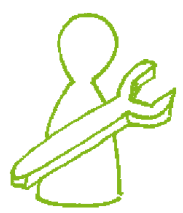

Verwenden Sie Etherpad für

- den spontanen Einsatz

- ohne großen Einrichtungsaufwand,

- $\quad$ ohne hohe Schwelle für den Zugang zur Zusammenarbeit

- $\quad$ ohne Ansprüche an Layout, Bilder etc.

Benutzen Sie Office-Programme, wenn Sie

- $\quad$ nur einzelne Dokumente gemeinsam bearbeiten

- ad hoc ein Dokument aufsetzen möchten

- Dokumente nachher exportieren und in ein Office-Programm importieren oder eine Sicherungskopie ablegen wollen

- Dokumente verschiedener Formate (Präsentation, Tabellenkalkulation etc.) nutzen möchten

Setzen Sie ein Wiki auf, wenn

- $\quad$ mehrere Dokumente miteinander vernetzt bearbeitet werden sollen

- Sie auch Multimediadateien ablegen möchten

- $\quad$ RSS-Feeds für Aktualisierungen verfügbar sein sollen

- das Exportieren keine große Rolle spielt

Um es auf den Punkt zu bringen: Für eine Ad-hoc-Arbeitsgruppe mit klarem Arbeitsauftrag wären Etherpad oder Office 2.0 die Mittel der Wahl, langfristig arbeitende Arbeits- und Projektgruppen tun gut daran, sich eine gut strukturierte Arbeitsplattform samt Ablage aufzubauen. Hier können auch neu hinzugekommene Mitglieder zurückverfolgen, was bereits erstellt und bearbeitet wurde. 